\title{
Philadelphia+ Chronic Myeloid Leukemia with CALR Mutation: A Case Report and Literature Review
}

\section{Seug Yun Yoon, $\mathrm{MD}^{1}$ \\ Sun Young Jeong, MD' \\ Changgon Kim, MD ${ }^{1}$ \\ Min-Young Lee, MD \\ Jieun Kim, MD, PhD² \\ Kyoung-Ha Kim, MD, PhD' \\ Namsu Lee, MD, PhD \\ Jong-Ho Won, MD, PhD ${ }^{1}$}

Myeloproliferative neoplasms (MPNs) are classified as chronic myeloid leukemia (CML) and Philadelphia chromosome-negative MPN. In MPN cases, the presence of a BCR-ABL1 translocation with a coexisting mutation is exceptionally rare. Herein, we report the first documented patient with CML harboring CALR mutation in Korea. A 33-year-old woman was referred to our hospital in February 2015 with splenomegaly, leukocytosis, and thrombocytosis. She was diagnosed with CML and started receiving nilotinib. In October 2015, a major molecular response was observed, but thrombocytosis persisted. A repeat bone marrow (BM) examination revealed no specific findings. However, as thrombocytosis worsened, we changed nilotinib to dasatinib. In May 2019, owing to persistent thrombocytosis, we repeated the BM examination and found CALR mutation (15.97\%) on the MPN-next generation sequencing (NGS) test. We then retrospectively performed repeat MPN-NGS testing using the BM aspirate sample obtained in 2015 and found CALR mutation (10.64\%).

${ }^{1}$ Division of Hematology and Medical Oncology, Department of Internal Medicine, ${ }^{2}$ Department of Laboratory Medicine, Soonchunhyang University Seoul Hospital, Seoul, Korea

Correspondence: Jong-Ho Won, $\mathrm{MD}, \mathrm{PhD}$ Division of Hematology and Medical Oncology, Department of Internal Medicine,

Soonchunhyang University Seoul Hospital, 59 Daesagwan-ro, Yongsan-gu,

Seoul 04401, Korea

Tel: 82-2-709-9203

Fax: 82-2-709-9200

E-mail: jhwon@schmc.ac.kr

Received November 18, 2019

Accepted January 13, 2020

Published Online January 15, 2020

Key words

Myeloproliferative disorder, Philadelphia chromosome, Calreticulin

\section{Introduction}

Myeloproliferative neoplasms (MPNs) are a group of disorders in which bone marrow (BM) stem cells show abnormal growth and reproduction. In MPNs, abnormal stem cells produce excess numbers of one or more types of blood cells. These abnormal cells do not function properly and can cause serious health problems unless properly treated and controlled. Chronic myeloid leukemia (CML) is a type of MPN characterized by the translocation between chromosome 9 and 22, which is called Philadelphia chromosome, thereby resulting in the BCR-ABL1 oncogene. According to 'The 2016 revision to the World Health Organization classification of myeloid neoplasm and acute leukemia', other classical MPNs can be diagnosed after CML is excluded. However, recent cases with the coexistence of $B C R-A B L 1$ and other MPN markers (i.e., $C A L R, J A K 2$, or MPL mutations) have been reported. Herein, we present a case of BCR-ABL1-positive CML with a CALR mutation in a patient with persistent 
thrombocytosis who maintained a major molecular response after treatment with a tyrosine kinase inhibitor (TKI).

\section{Case Report}

A 33-year-old woman was referred to our hospital in February 2015 owing to an abdominal mass and abnormal laboratory values. Abdominal ultrasonography revealed splenomegaly $(22 \mathrm{~cm})$, and laboratory examinations revealed a white blood cell (WBC) count of $107,600 / \mathrm{mm}^{3}$ and a platelet (PLT) count of $906,000 / \mathrm{mm}^{3}$. BM examination revealed a marrow cellularity of $90 \%$, with markedly increased megakaryocytes. Reticulin staining indicated grade 2-3 fibrosis. The karyotype was 45,XX,t(9;22)(q34;q11.2), der(13;14)(q10;q10)[15]. In case of der(13;14)(q10;q10), it was found in all cells continuously after treatment and presumed to be germline mutation. Fluorescence in situ hybridization analysis showed that $99.2 \%$ of 500 cells were positive for $t(9 ; 22)$, and real-time quantitative polymerase chain reaction showed that the transcript level for BCR-ABL1/ ABL1 ${ }^{\text {IS }}$ (international scale [IS]) was $25.603 \%$. JAK2 V617F was negative on conventional screening. Testing for CALR mutations was not yet available. Accordingly, the patient started receiving nilotinib. After 6 months of treatment with nilotinib, she achieved a major molecular response. In October 2015, she developed persist-

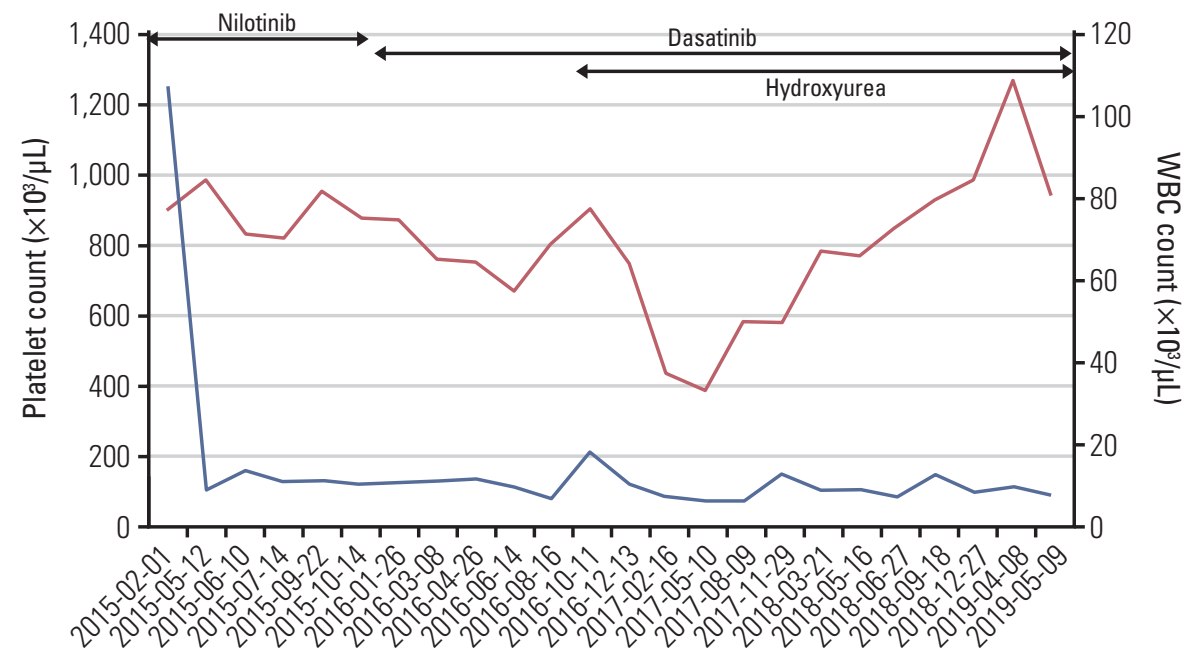

Fig. 1. White blood cell (WBC) and platelet counts during follow-up of a patient with chronic myeloid leukemia over the course of treatment.

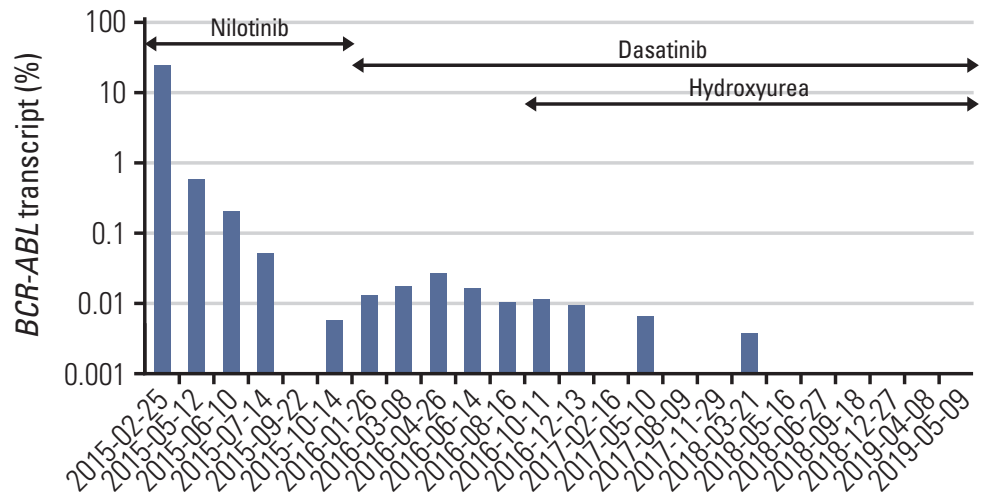

Fig. 2. $B C R-A B L 1$ transcript levels during follow-up of a patient with chronic myeloid leukemia over the course of treatment. 


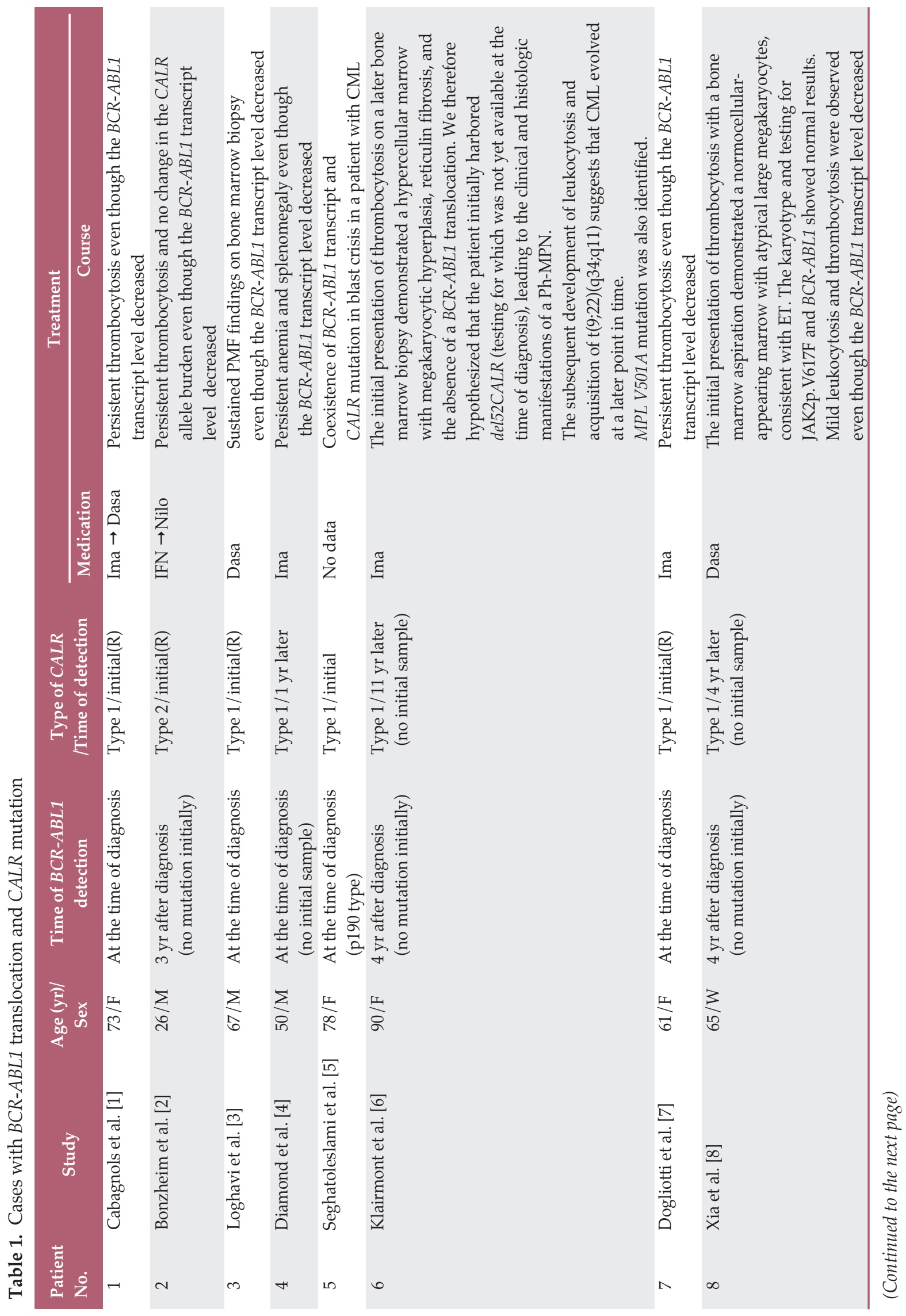




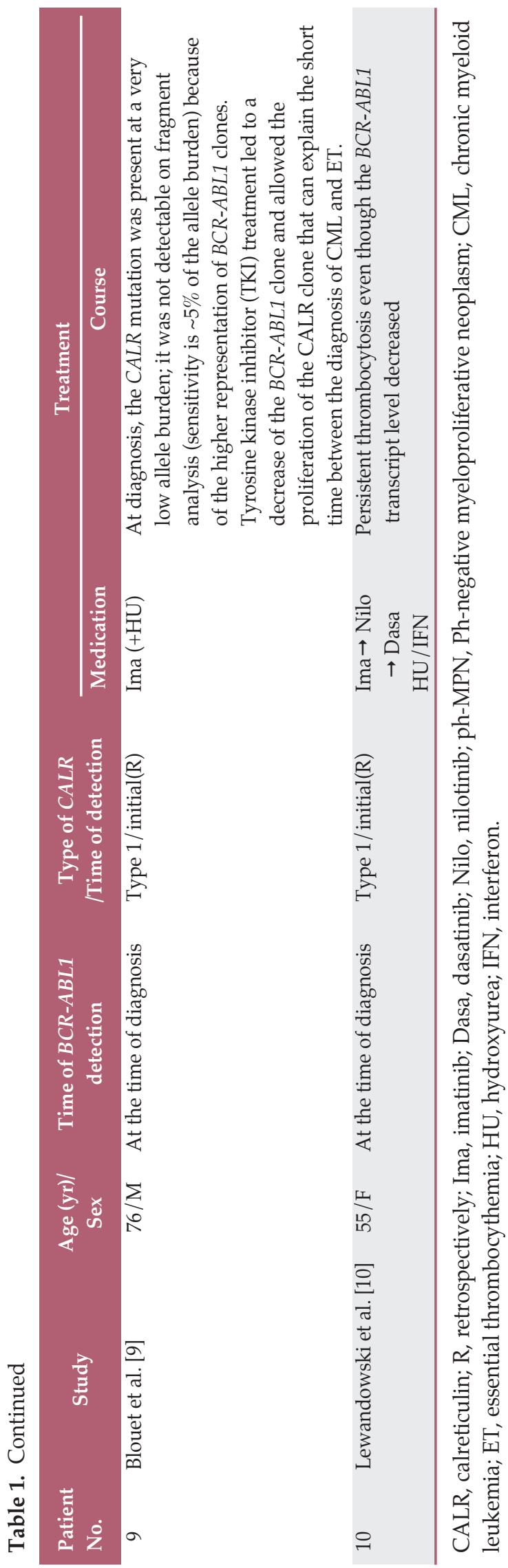

ent thrombocytosis. A repeat BM examination did not show any specific findings. The karyotype was $46, \mathrm{XX}$, der(13;14) (q10;q10)[13]. However, as thrombocytosis worsened (PLT count, 1,006,000/ $\mathrm{mm}^{3}$ ) and splenomegaly persisted, we changed nilotinib to dasatinib. We then added hydroxyurea as part of the treatment, after which the thrombocytosis was controlled slightly but it worsened subsequently (Figs. 1 and 2). In May 2019, follow-up BM examination was performed, and we used the BM aspiration sample for MPN-next-generation sequencing (NGS): the patient had CALR mutation (c.1099_1136delinsAGGT), with an allele frequency of $15.97 \%$, and TET2 mutation (c.3409+1G>A), with an allele frequency of $7.83 \%$. We then retrospectively performed repeat MPNNGS testing using the BM aspiration sample obtained in 2015. We found that CALR mutation had been presence since 2015 , with an allele frequency of $10.64 \%$, but no TET2 mutation was observed. We reported a case of CALR-positive CML with persistent thrombocytosis during TKI treatment.

The study protocol was approved by the Institutional Review Board of the Soonchunhyang University Seoul Hospital (No. 2020-01-012). The study patient was informed as the purpose of the study and provided her consent.

\section{Discussion}

CALR mutation was first recognized as a somatic mutation in patients with MPN who had no mutation in either JAK2 or MPL in 2013 [11]. CALR mutation is observed in approximately $70 \%$ of patients with essential thrombocythemia (ET) or primary myelofibrosis who did not carry a mutation in either JAK2 or MPL [11,12]. CALR-mutant MPNs are characterized by a gene signature associated with activating JAK2 signaling, and CALR-mutants activate the JAK/STAT pathway through MPL, leading to excessive PLT production [13]. More than 50 different mutations in CALR have been described, but a 52 base-pair deletion (type 1) or a 5 base-pair insertion (type 2) account for more than $80 \%$ of mutations [13]. Pietra et al. [14] showed an association between CALR type 1 mutations and a significant increase in the risk of myelofibrotic transformation among ET cases, whereas type 2 mutations were preferentially associated with an ET phenotype, low risk of thrombosis despite a very high PLT count, and an indolent clinical course.

The presence of a $B C R-A B L 1$ translocation with a coexisting mutation in CALR is exceptionally rare. A total of 10 cases have been reported since the first report was published in 2015, and the current report is the first in Korea (Table 1). In most cases, CALR mutation and BCR-ABL1 translocation were synchronously identified or $B C R-A B L 1$ translocation 
was detected after $C A L R$ mutation. Otherwise, the presence of CALR mutation was not confirmed, but there was a case where the diagnosis was changed to CML on the basis of the findings of ET. To the best of our knowledge, there has been no report of $C A L R$ mutation after CML diagnosis. TKIs were ineffective for $C A L R$-mutated clones, as shown in previous studies. Accordingly, physicians should be careful while changing the treatment of patients with CML who achieve a molecular response but do not obtain a hematologic response. Additional molecular mutations may be identified in patients with CML with the recent widespread use of NGS testing. A recent study showed the presence of molecular aberrations other than JAK2 V617F and BCR-ABL1 in 56\% of patients with MPN, including mutations in TET2, ASXL1, RUNX1, CBL, DNMT3A, PHF6, SF3B1, and TP53 [15]. In particular, TET2 is a putative tumor suppressor gene located on chromosome 4q24. Only a few studies revealed TET2 mutations in patients with MPN. However, there is no consensus regarding the exact role of TET2. In the current case, the relevance of identifying a new TET2 mutation during the course of the disease is unclear. Although patients with CML with $B C R-A B L 1$ translocation have additional molecular aberrations, they are still diagnosed with CML on the basis of the current diagnostic criteria. However, further studies are needed regarding the diagnosis, treatment, and prognosis of these patients.

\section{Conflicts of Interest}

Conflict of interest relevant to this article was not reported.

\section{Acknowledgments}

This study was supported by the Soonchunhyang University Research Fund. We would like to thank Editage (www.editage. co.kr) for English language editing.

\section{References}

1. Cabagnols X, Cayuela JM, Vainchenker W. A CALR mutation preceding BCR-ABL1 in an atypical myeloproliferative neoplasm. N Engl J Med. 2015;372:688-90.

2. Bonzheim I, Mankel B, Klapthor P, Schmidt J, Hinrichsen T, Wachter $\mathrm{O}$, et al. CALR-mutated essential thrombocythemia evolving to chronic myeloid leukemia with coexistent CALR mutation and BCR-ABL translocation. Blood. 2015;125:2309-11.

3. Loghavi S, Pemmaraju N, Kanagal-Shamanna R, Mehrotra M, Medeiros LJ, Luthra R, et al. Insights from response to tyrosine kinase inhibitor therapy in a rare myeloproliferative neoplasm with CALR mutation and BCR-ABL1. Blood. 2015;125:3360-3.

4. Diamond JM, de Almeida AM, Belo HJ, da Costa MP, Cabecadas JM, Abecasis MM. CALR-mutated primary myelofibrosis evolving to chronic myeloid leukemia with both CALR mutation and BCR-ABL1 fusion gene. Ann Hematol. 2016;95: 2101-4.

5. Seghatoleslami M, Ketabchi N, Ordo A, Asl JM, Golchin N, Saki N. Coexistence of p190 BCR/ABL transcript and CALR 52-bp deletion in chronic myeloid leukemia blast crisis: a case report. Mediterr J Hematol Infect Dis. 2016;8:e2016002.

6. Klairmont MM, Cheng J, Schwartzberg L, Ho HH, Gradowski JF. Chronic myeloid leukemia, BCR-ABL1-positive with CALR and MPL mutations. Int J Lab Hematol. 2018;40:e41-2.

7. Dogliotti I, Fava C, Serra A, Gottardi E, Daraio F, Carnuccio F, et al. CALR-positive myeloproliferative disorder in a patient with Ph-positive chronic myeloid leukemia in durable treatment-free remission: a case report. Stem Cell Investig. 2017; 4:57.

8. Xia D, Hsi ED, Dal Cin P, Hasserjian RP. Composite chronic myeloid leukemia and essential thrombocythemia with BCR-
ABL1 fusion and CALR mutation. Am J Hematol. 2019;94:504-5. 9. Blouet A, Rousselet MC, Le Bris Y, Ribourtout B, Bouvier A, Cottin L, et al. Imatinib treatment of chronic myeloid leukemia reveals a preexisting CALR-mutated essential thrombocythemia. Hemasphere. 2018;2:e29.

10. Lewandowski K, Gniot M, Wojtaszewska M, Kandula Z, Becht R, Paczkowska E, et al. Coexistence of JAK2 or CALR mutation is a rare but clinically important event in chronic myeloid leukemia patients treated with tyrosine kinase inhibitors. Int J Lab Hematol. 2018;40:366-71.

11. Nangalia J, Massie CE, Baxter EJ, Nice FL, Gundem G, Wedge DC, et al. Somatic CALR mutations in myeloproliferative neoplasms with nonmutated JAK2. N Engl J Med. 2013;369:2391405.

12. Tefferi A, Lasho TL, Finke CM, Knudson RA, Ketterling R, Hanson $\mathrm{CH}$, et al. CALR vs JAK2 vs MPL-mutated or triplenegative myelofibrosis: clinical, cytogenetic and molecular comparisons. Leukemia. 2014;28:1472-7.

13. Cazzola M, Kralovics R. From Janus kinase 2 to calreticulin: the clinically relevant genomic landscape of myeloproliferative neoplasms. Blood. 2014;123:3714-9.

14. Pietra D, Rumi E, Ferretti VV, Di Buduo CA, Milanesi C, Cavalloni $C$, et al. Differential clinical effects of different mutation subtypes in CALR-mutant myeloproliferative neoplasms. Leukemia. 2016;30:431-8.

15. Martin-Cabrera P, Haferlach C, Kern W, Schnittger S, Haferlach T. BCR-ABL1-positive and JAK2 V617F-positive clones in 23 patients with both aberrations reveal biologic and clinical importance. Br J Haematol. 2017;176:135-9. 